\title{
Second Degree Generalized Successive Over Relaxation Method for Solving System of Linear Equations
}

\author{
Firew Hailu ${ }^{1}$, Genanew Gofe Gonfa ${ }^{2}$ and Hailu Muleta Chemeda ${ }^{3 *}$ \\ ${ }^{1}$ Department of Mathematics, Bonga College of Teachers' Education, Bonga, Ethiopia \\ ${ }^{2}$ Department of Mathematics, College of Natural Sciences, Salale University, Salale, Ethiopia \\ ${ }^{3}$ Department of Mathematics, College of Natural Sciences, Jimma University, Jimma, Ethiopia \\ (*muletah@gmail.com).
}

\begin{abstract}
In this paper, a second degree generalized successive over relaxation iterative method for solving system of linear equations based on the decomposition $A=D_{m}+L_{m}+U_{m}$ is presented and the convergence properties of the proposed method are discussed. Two numerical examples are considered to show the efficiency of the proposed method. The results presented in tables show that the Second Degree Generalized Successive Over Relaxation Iterative method is more efficient than the other methods considered based on number of iterations, computational running time and accuracy.
\end{abstract}

Keywords: Second Degree, Generalized Gauss Seidel, Successive over relaxation, Convergence.

\section{INTRODUCTION}

Consider a system of linear equations

$$
A x=b
$$

Where, $A$ is an $n x n$ nonsingular coefficient matrix, $b$ is a column vector and $x$ is solution vector to be determined.

Splitting the matrix $A$ as in Young (1972); and Genanew Gofe (2016),

$$
A=D_{m}+L_{m}+U_{m}
$$

Where, $D_{m}=\left(d_{i j}\right)$ be a banded matrix with band length $2 \mathrm{~m}+1$ is defined as

$$
d_{i j}=\left\{\begin{array}{l}
a_{i j},|j-i| \leq m \\
0 ; \text { othetwise }
\end{array}\right.
$$

$L_{m}$ and $U_{m}$ are strictly lower and upper triangular parts of $A-D_{m}$, respectively, and they are defined as follows: 


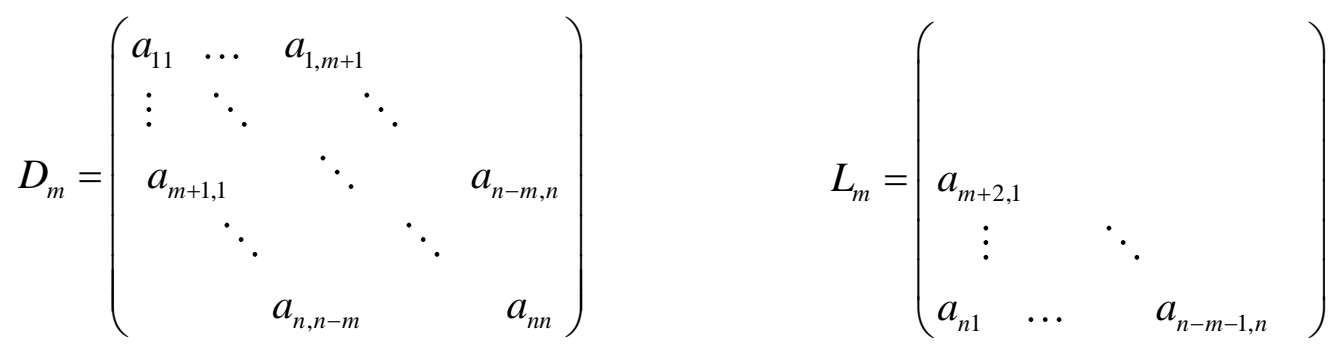

and

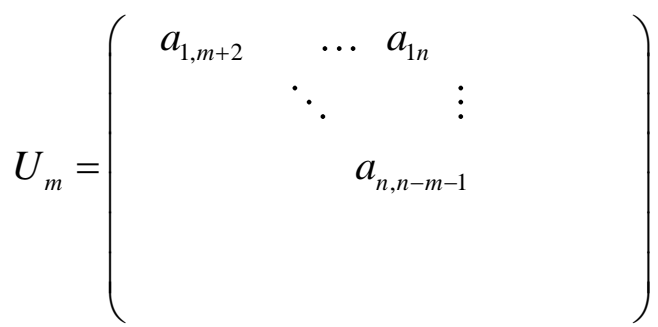

The linear stationary first degree iteration method defined by Young (1972) is given by

$$
x^{(k+1)}=G_{1} x^{(k)}+C
$$

Here, $G_{1}$ is an iteration matrix and $C$ is the corresponding column vector.

The first degree Jacobi, Gauss Seidel and Successive over relaxation methods for solving equation (1) can be obtained in the form of

$$
\begin{aligned}
& x^{(k+1)}=-\left(D_{m}\right)^{-1}\left(L_{m}+U_{m}\right) x^{(k)}+D_{m}^{-1} b \\
& x^{(k+1)}=-\left(D_{m}+L_{m}\right)^{-1} U_{m} x^{(k)}+\left(D_{m}+L_{m}\right)^{-1} b \\
& x^{k+1}=\left(D_{m}+\omega L_{m}\right)^{-1}\left((1-\omega) D_{m}-\omega U_{m}\right) x^{k}+\omega\left(D_{m}+\omega L_{m}\right)^{-1} b
\end{aligned}
$$

, respectively, when $m=0$.

The First Degree Generalized Successive over relaxation for solving equation (1) reformulated by Davod (2007) is given by

$$
\begin{aligned}
& x^{(k+1)}=\left(D_{m}+\omega L_{m}\right)^{-1}\left((1-\omega) D_{\mathrm{m}}-\omega \mathrm{U}_{\mathrm{m}}\right) \mathrm{x}^{(\mathrm{k})}+\left(D_{\mathrm{m}}+\omega \mathrm{L}_{\mathrm{m}}\right)^{-1} \omega \mathrm{b} \\
& x^{(k+1)}=B_{G S O R} x^{(k)}+C_{s} \quad \text { where, } k=0,1,2, \ldots \\
& B_{G S O R}=\left(D_{m}+\omega L_{m}\right)^{-1}\left((1-\omega) D_{\mathrm{m}}-\omega \mathrm{U}_{\mathrm{m}}\right) \\
& C_{s}=\left(D_{\mathrm{m}}+\omega \mathrm{L}_{\mathrm{m}}\right)^{-1} \omega \mathrm{b}
\end{aligned}
$$


Equation (5) is the Generalized Successive over relaxation iteration matrix and equation (6) is its iteration vector.

Definition1. A matrix $A$ is said to be reducible if there exists a permutation matrix $\mathrm{P}$ such that $P A P^{T}$ is a block upper triangular matrix; otherwise it is an irreducible.

Definition2. A matrix $A$ is said to be strictly diagonally dominant (SDD) if

$$
\left|a_{i i}\right|>\sum_{j=1, j \neq i}^{n}\left|a_{i j}\right|, \quad i=1,2, \ldots, n .
$$

and is said to be weakly diagonally dominant (WDD) if

$$
\left|a_{i i}\right| \geq \sum_{j=1, j \neq i}^{n}\left|a_{i j}\right|, \quad i=1,2, \ldots, n .
$$

Definition3. A matrix $A$ is said to be irreducibly weakly diagonally dominant (IWDD) if $A$ is WDD and irreducible.

Definition4. A real matrix $A$ is said to be positive definite or positive real if $(A x, x)>0$, $\forall x \in \mathfrak{R}^{N}, x \neq 0$.

Theorem1. Let $A$ and $D_{m}$ be SDD matrices. Then for every $0<\omega<2$, the GSOR method converges with any initial guess $x_{0}$. The detail of the proof is given by Davod (2007).

Theorem2. Let $0<\omega \leq 1$ and $A$ be $I W D D$ matrix and $D_{m}$ be irreducible matrix. Then the associated GSOR method is convergent for every initial guess $x_{0}$. One can refer Davod (2007) for the detail of the proof.

\section{SECOND DEGREE GENERALIZED SUCCESSIVE OVER RELAXATION METHOD}

Tesfaye Kebede (2016) defined the linear stationary second degree method as

$$
x^{(k+1)}=x^{(k)}+d\left(x^{(k)}-x^{(k-1)}\right)+e\left(x^{(k+1)}-x^{(k)}\right)
$$

Substituting equation (3) into the right hand side of equation (7), we obtain

$$
\begin{aligned}
& x^{(k+1)}=x^{(k)}+d\left(x^{(k)}-x^{(k-1)}\right)+e\left(G_{1} x^{(k)}+C-x^{(k)}\right) \\
& x^{(k+1)}=x^{(k)}+d x^{(k)}-e x^{(k-1)}+e G_{1} x^{(k)}+e C-e x^{(k)} \\
& x^{(k+1)}=\left[(1+d-e) I+e G_{1}\right] x^{(k)}-d x^{(k-1)}+e C
\end{aligned}
$$




$$
x^{(k+1)}=G x^{(k)}+H x^{(k-1)}+l
$$

Where, $G=(1+d-e) I+e G_{1} ; \quad H=-d I ; \quad l=e C$, which is completely consistent for any constants $d$ and $e$ such that $e \neq 0$.

The Second Degree Generalized SOR iteration method is denoted by

$$
x^{(k+1)}=G x^{(k)}+H x^{(k-1)}+l
$$

Where,

$$
\begin{aligned}
G & =(1+d-e) I+e G_{s} \\
H & =-d I \\
l & =e C_{s}
\end{aligned}
$$

$G_{s}$ is the iteration matrix of generalized successive over relaxation iterative method and $C_{s}$ is its corresponding column vector. Hence $G_{s}=B_{G S O R}=\left(D_{m}+\omega L_{m}\right)^{-1}\left((1-\omega) D_{\mathrm{m}}-\omega \mathrm{U}_{\mathrm{m}}\right)$, and $\quad C_{s}=\left(D_{\mathrm{m}}+\omega \mathrm{L}_{\mathrm{m}}\right)^{-1} \omega \mathrm{b}$.

Using the idea of Golub and Varga (1961), equation (8) can be written in the matrix form as

$$
\left(\begin{array}{c}
x^{(k)} \\
x^{(k+1)}
\end{array}\right)=\left(\begin{array}{cc}
0 & I \\
H & G
\end{array}\right)\left(\begin{array}{c}
x^{(k-1)} \\
x^{(k)}
\end{array}\right)+\left(\begin{array}{l}
0 \\
l
\end{array}\right)
$$

The necessary and sufficient conditions for convergence of the method is that the spectral radius of $G$ must be less than unity in magnitude for any $x^{(0)}$ and $x^{(1)}$, Where, $G=\left(\begin{array}{cc}0 & I \\ H & G\end{array}\right)$ is the second degree iteration matrix.

Let $\lambda$ be the spectral radius of $G$.

$\Rightarrow G x=\lambda x$

Let $x=\left(\begin{array}{l}u_{1} \\ u_{2}\end{array}\right), \quad x \neq 0$. 
$\Rightarrow u_{2}=\lambda u_{1}$ and $\mathrm{Hu}_{1}+G u_{2}=\lambda u_{2} I$

but $u_{2}=\lambda u_{1}$, substituting $u_{2}$ into Eq. (12), we get

$\Rightarrow H u_{1}+G u_{2}=\lambda^{2} u_{1} I$

$\Rightarrow H u_{1}+G u_{2}-\lambda^{2} u_{1} I=0, \quad 0 \varepsilon R^{n \times n}$.

$\Rightarrow u_{1}\left(H+G-\lambda^{2} I\right)=0$

$\Rightarrow H+G-\lambda^{2} I=0, \quad$ since $u_{1} \neq 0$

Substituting $G=(1+d+e) I+e G_{s}, H=-d I$ and $l=e C_{s}$ into eq. (13), we obtain

$\lambda^{2} I-\left[(1+d-e) I+e G_{s}\right] \lambda+d I=0$

$\Rightarrow \lambda^{2} I-(1+d-e) \lambda I-e G_{s} \lambda+d I=0$

$\Rightarrow-e \lambda\left[\frac{-\lambda^{2} I}{e \lambda}+\frac{(1+d-e) \lambda I}{e \lambda}+\frac{e G_{s} \lambda}{e \lambda}-\frac{d I}{e \lambda}\right]=0$

$\frac{-\lambda^{2} I}{e \lambda}+\frac{(1+d-e) \lambda I}{e \lambda}+\frac{e G_{s} \lambda}{e \lambda}-\frac{d I}{e \lambda}=0, \quad$ since $-e \lambda \neq 0$.

$\frac{-\lambda I}{e}+\frac{(1+d-e) I}{e}+G_{s}-\frac{d I}{e \lambda}=0, \quad 0 \varepsilon R^{n \times n}$

If $\lambda$ is the eigenvalue of $G$ and $\mu$ is the eigenvalue of $G_{s}$, then

$$
\begin{gathered}
\operatorname{det}\left(G_{s}+\frac{(1+d-e) I}{e}-\frac{\left(d+\lambda^{2}\right) I}{e \lambda}\right)=0 \text { has solution } \\
\mu+\frac{(1+d-e)}{e}-\frac{\left(d+\lambda^{2}\right)}{e \lambda}=O, O \varepsilon R .
\end{gathered}
$$

As discussed in Young (1972), if we let

$$
\lambda=r e^{i \theta}=r(\cos \theta+i \sin \theta)
$$

The Spectral radius of $G=\max \left|\lambda_{i}\right|=\left|r e^{i \theta}\right|=|r|$.

So substituting equation (15) into equation (14), we obtain

$$
\mu+\frac{1+d-e}{e}=\frac{\left(r e^{i \theta}\right)^{2}+d}{e r e^{i \theta}}=\frac{(r \cos \theta+i r \sin \theta)^{2}+d}{e r(\cos \theta+i \sin \theta)}
$$

After simplifying and collecting like terms, we get 


$$
\mu=\frac{e-1-d}{e}+\left(\frac{r^{2}+d}{e r}\right) \cos \theta+i\left(\frac{r^{2}-d}{e r}\right) \sin \theta
$$

From equation (16), we have

$$
\operatorname{Re}(\mu)=\frac{e-1-d}{e}+\left(\frac{r^{2}+d}{e r}\right) \cos \theta, \text { and } \operatorname{Im}(\mu)=\left(\frac{r^{2}-d}{e r}\right) \sin \theta
$$

From $\operatorname{Re}(\mu)$, we get

$$
\operatorname{Re}(\mu)+\frac{1+d-e}{e}=\left(\frac{r^{2}+d}{e r}\right) \cos \theta
$$

Solving for $\cos \theta$, we have $\cos \theta=\frac{\operatorname{Re}(\mu)+\frac{1+d-e}{e}}{\left(\frac{r^{2}+d}{e r}\right)}$

$$
(\cos \theta)^{2}=\left(\frac{\operatorname{Re}(\mu)+\frac{1+d-e}{e}}{\left(\frac{r^{2}+d}{e r}\right)}\right)^{2}
$$

From $\operatorname{Im}(\mu)=\left(\frac{r^{2}-d}{e r}\right) \sin \theta$, we obtain $\sin \theta=\frac{\operatorname{Im}(\mu)}{\left(\frac{r^{2}-d}{e r}\right)}$.

$$
(\sin \theta)^{2}=\left(\frac{\operatorname{Im}(\mu)}{\frac{r^{2}-d}{e r}}\right)^{2}
$$

Adding equations (17) and (18), we obtain

$$
\left(\frac{\operatorname{Re}(\mu)+\frac{1+d-e}{e}}{\frac{r^{2}+d}{e r}}\right)^{2}+\left(\frac{\operatorname{Im}(\mu)}{\frac{r^{2}-d}{e r}}\right)^{2}=1 \quad\left(\text { Since } \sin ^{2} \theta+\cos ^{2} \theta=1\right)
$$

If $\mu$ is real and varies over the range of $\alpha \leq \mu \leq \beta<1$, then the choices of $d$ and $e$ which minimize the spectral radius of $G$ are given by 


$$
d=\hat{\omega}_{b}-1, \quad e=\frac{2 \hat{\omega}_{b}}{2-(\beta+\alpha)}
$$

Where, $\quad \hat{\omega}_{b}=\frac{2}{\left(1+\sqrt{\left(1-\sigma^{2}\right)}\right)} \quad$ and $\quad \sigma=\frac{(\beta-\alpha)}{(2-(\beta+\alpha))}$

The corresponding values of the spectral radius of $\hat{G}$ is

$$
\rho(\hat{G})=d^{1 / 2}=\sqrt{\hat{\omega}_{b}-1}
$$

Thus, with these choices of $d$ and $e$, we have

$$
\begin{gathered}
G=(1+d-e) I+e G_{s}=\hat{\omega}_{b}\left(\frac{2 G_{s}}{2-(\beta+\alpha)}-\frac{(\beta+\alpha) I}{2-(\beta+\alpha)}\right) \\
H=-d I=\left(1-\hat{\omega}_{b}\right) I \\
l=e C_{s}=\frac{2 \hat{\omega}_{b} C_{s}}{2-(\beta+\alpha)}
\end{gathered}
$$

Hence, equation (8) becomes

$$
x^{(k+1)}=\hat{\omega}_{b}\left(\frac{2 G_{s}}{2-(\beta+\alpha)}-\frac{(\beta+\alpha) I}{2-(\beta+\alpha)}\right) x^{(k)}+\left(1-\hat{\omega}_{b}\right) x^{(k-1)}+\frac{2 \hat{\omega}_{b} C_{s}}{2-(\beta+\alpha)}
$$

According to Young (1972); and David (1994), if $A$ is symmetric positive definite (SPD) matrix, then $A$ has real nonnegative eigenvalues and we can apply the second degree iterative method using $\alpha=0$ and $\beta=\bar{\mu}$.

$$
\sigma=\frac{\beta-\alpha}{2-(\beta+\alpha)}=\frac{\bar{\mu}}{2-\bar{\mu}}
$$

Where, $\bar{\mu}$ is the spectral radius of the Generalized SOR iteration matrix.

$$
\begin{aligned}
& \hat{\omega}_{b}=\frac{2(2-\bar{\mu})}{2-\bar{\mu}+2 \sqrt{1-\bar{\mu}}}=\frac{2(2-\bar{\mu})}{(1+\sqrt{1-\bar{\mu}})^{2}} \\
& d=\hat{\omega}_{b}-1=\frac{2(2-\bar{\mu})}{(1+\sqrt{1-\bar{\mu}})^{2}}-1=\frac{2(2-\bar{\mu})-(1+\sqrt{1-\bar{\mu}})^{2}}{(1+\sqrt{1-\bar{\mu}})^{2}}
\end{aligned}
$$




$$
=\left(\frac{2-\bar{\mu}-2 \sqrt{1-\bar{\mu}}}{(1+\sqrt{1-\bar{\mu}})^{2}}\right)\left(\frac{2-\bar{\mu}+2 \sqrt{1-\bar{\mu}}}{2-\bar{\mu}+2 \sqrt{1-\bar{\mu}}}\right) \quad \frac{(2-\bar{\mu})^{2}-(2 \sqrt{1-\bar{\mu}})^{2}}{(1+\sqrt{1-\bar{\mu}})^{4}}=\frac{\bar{\mu}^{2}}{(1+\sqrt{1-\bar{\mu}})^{4}}
$$

Thus, $\quad d=\frac{\bar{\mu}^{2}}{(1+\sqrt{1-\bar{\mu}})^{4}}$

From equation (20), we have

$$
\begin{aligned}
& e=\frac{2 \sigma \hat{\omega}_{b}}{(\beta-\alpha)}=\frac{2 \hat{\omega}_{b}}{2-(\beta+\alpha)}=\frac{2}{2-\bar{\mu}}\left(\frac{2(2-\bar{\mu})}{(1+\sqrt{1-\bar{\mu}})^{2}}\right)=\frac{4}{(1+\sqrt{1-\bar{\mu}})^{2}} \\
& \rho(G)=d^{1 / 2}=\sqrt{\hat{\omega}_{b}-1}=\sqrt{\frac{\bar{\mu}^{2}}{(1+\sqrt{1-\bar{\mu}})^{4}}}=\frac{\bar{\mu}}{(1+\sqrt{1-\bar{\mu}})^{2}}
\end{aligned}
$$

Therefore, the Second Degree Generalized SOR, according to equation (8), is given by

$$
x^{(k+1)}=\hat{\omega}_{b}\left(\frac{2 B_{G S O R}}{2-(\beta+\alpha)}-\frac{(\beta+\alpha) I}{2-(\beta+\alpha)}\right) x^{(k)}+\left(1-\hat{\omega}_{b}\right) x^{(k-1)}+\frac{2 \hat{\omega}_{b} C_{s}}{2-(\beta+\alpha)}
$$

Letting $\alpha=0$ and $\beta=\bar{\mu}$, we get

$$
x^{(k+1)}=\hat{\omega}_{b}\left(\frac{2 B_{G S O R}}{2-\bar{\mu}}-\frac{\bar{\mu} I}{2-\bar{\mu}}\right) x^{(k)}+\left(1-\hat{\omega}_{b}\right) x^{(k-1)}+\frac{2 \hat{\omega}_{b} C_{s}}{2-\bar{\mu}}
$$

Where, $\quad \hat{\omega}_{b}=\frac{4-2 \bar{\mu}}{(1+\sqrt{1-\bar{\mu}})^{2}}$

\subsection{Numerical Examples}

To illustrate the feasibility and efficiency of the present method when employed to solve system of linear equations, we used two systems of linear equations. All the numerical experiments presented in this section are made in the same condition, using the same problems, using the same processor, memory size and operating system. The processor used is Intel(R) core (TM) i3- 
31110M CPU @2.40GHz 2.40GHz with 4GM memory (RAM) with 64 bits operating system (Window 7 home premium). The language program used is MATLAB version 7.60(R2008a). The major factors considered in comparing different numerical methods are i) the accuracy of the numerical solutions, and ii) its computational time (Bedet et al., 1975; and Gananew Gofe, 2016). It should also be noted that there are other factors to be considered such as stability, versatility, proof against run-time error, and so on which are being considered in most of the MATLAB built in routines (Yang et al., 2005; and Kalambi, 2008). Data about iteration number and computational time (in seconds) obtained using SOR, GSOR and SDGSOR is used for analysis of the result. 1. Solve the system of linear equations considered by Noreen (2012) by SOR, GSOR and SDGSOR iterative methods.

$$
\begin{gathered}
4 x_{1}-x_{2}-x_{3}=0.5 \\
-x_{1}+4 x_{2}-x_{4}=1.3 \\
-x_{1}+4 x_{3}-x_{4}=1 \\
-x_{2}-x_{3}+4 x_{4}=1.8
\end{gathered}
$$

2. Solve the system of linear equations considered by Noreen (2012) by SOR, GSOR and SDGSOR iterative methods

$$
\begin{array}{lcc}
4 x_{1}-x_{2}-x_{4} & = & 0.707 \\
-x_{1}+4 x_{2}-x_{3}-x_{5} & = & 1 \\
-x_{2}+4 x_{3}-x_{6} & = & 0.707 \\
-x_{1}+4 x_{4}-x_{5}-x_{7} & = & 0 \\
-x_{2}-x_{4}+4 x_{5}-x_{6}-x_{8} & = & 0 \\
-x_{3}-x_{5}+4 x_{6}-x_{9} & = & 0 \\
-x_{4}+4 x_{7}-x_{8} & = & 0 \\
-x_{5}-x_{7}+4 x_{8}-x_{9} & = & 0 \\
-x_{6}-x_{8}+4 x_{9} & = & 0
\end{array}
$$

The above examples are solved numerically by using SOR, GSOR and SGSOR and the results are presented in tables $1,2,3$ and 4 .

Table 1. Spectral radii of SOR, GSOR and SDGSOR iterative methods when $m=1$.

\begin{tabular}{|llcl|}
\hline Method & SOR $(\omega=1.09)$ & GSOR $(\omega=1.035)$ & SDGSOR $(\omega=1.035)$ \\
\hline Spectral radius & 0.3745535796227922 & 0.03499999999999992 & 0.034999999999999992 \\
\hline
\end{tabular}


Table 2. Solution of example 1 by SOR, GSOR and SDGSOR iterative methods when $m=1$.

\begin{tabular}{|lcclccc|}
\hline Method & $\begin{array}{l}\text { Relaxation } \\
\text { factor, } \omega\end{array}$ & $\begin{array}{l}\text { Iteration } \\
\text { number }\end{array}$ & $\begin{array}{l}\text { Approximate } \\
\text { solution, } \boldsymbol{y}\end{array}$ & Exact solution, $\boldsymbol{x}$ & $\begin{array}{c}\text { Absolute Error } \\
|\boldsymbol{x}-\boldsymbol{y}|\end{array}$ & $\begin{array}{c}\boldsymbol{C P U} \\
\text { time }\end{array}$ \\
\hline SOR & 1.090 & 7 & 0.4125003284401 & 0.4125000000000 & $3.28440078135 \mathrm{e}-7$ & \\
& & & 0.6125000070803 & 0.6124999999999 & $7.08026659524 \mathrm{e}-9$ & \\
& & & 0.5375000034930 & 0.5374999999999 & $3.49303985736 \mathrm{e}-9$ & 0.0035 \\
& & & 0.7374999953831 & 0.7374999999999 & $4.61693638876 \mathrm{e}-9$ & \\
\hline GSOR & 1.035 & 5 & 0.4125002956384 & 0.4125000000000 & $2.95638418413 \mathrm{e}-7$ & \\
& & & 0.6125003299105 & 0.6124999999999 & $3.29910521568 \mathrm{e}-7$ & \\
& & & 0.5375000661756 & 0.5374999999999 & $6.61756276443 \mathrm{e}-8$ & 0.00171 \\
& & & 0.7375000776497 & 0.7374999999999 & $7.76496594801 \mathrm{e}-8$ & \\
\hline SDGSOR & 1.035 & 5 & 0.4124999986993 & 0.4125000000000 & $1.30068600335 \mathrm{e}-9$ & \\
& & & 0.6124999913962 & 0.6124999999999 & $8.60384374857 \mathrm{e}-9$ & \\
& & & 0.5374999997450 & 0.5374999999999 & $2.5497348677 \mathrm{e}-10$ & 0.00171 \\
& & & 0.7374999983619 & 0.7374999999999 & $1.63813707044 \mathrm{e}-9$ & \\
\hline
\end{tabular}

Table 3. Spectral radii of SOR, GSOR and SDGSOR iterative methods when $m=1$.

\begin{tabular}{|llrl|}
\hline Method & SOR $(\omega=1.09)$ & GSOR $(\omega=1.035)$ & SDGSOR $(\omega=1.035)$ \\
\hline Spectral radius & 0.6579610242530591 & 0.2947201498259546 & 0.075810369327300 \\
\hline
\end{tabular}

Table 4. Solution of example 2 by SOR, GSOR and SDGSOR iterative methods when $m=1$.

\begin{tabular}{|c|c|c|c|c|c|c|}
\hline Method & $\begin{array}{c}\text { Relaxation } \\
\text { factor, } \omega\end{array}$ & $\begin{array}{c}\text { Iteration } \\
\text { number }\end{array}$ & $\begin{array}{l}\text { Approximate } \\
\text { solution, } y\end{array}$ & Exact solution, $x$ & $\begin{array}{c}\text { Absolute Error } \\
|x-y|\end{array}$ & $\begin{array}{l}\text { CPU } \\
\text { time }\end{array}$ \\
\hline \multirow[t]{9}{*}{ SOR } & 1.090 & 15 & 0.337003902050 & 0.337005127298 & $1.22524839429 \mathrm{e}-6$ & 0.00905 \\
\hline & & & 0.485662793979 & 0.485664073550 & $1.27957121454 \mathrm{e}-6$ & \\
\hline & & & 0.381818616783 & 0.381819483734 & $8.66951305967 \mathrm{e}-7$ & \\
\hline & & & 0.155355351684 & 0.155356435644 & $1.08395943750 \mathrm{e}-6$ & \\
\hline & & & 0.223830615589 & 0.223831683168 & $1.06757949442 \mathrm{e}-6$ & \\
\hline & & & 0.167306373237 & 0.167306930693 & $5.57455939854 \mathrm{e}-7$ & \\
\hline & & & 0.060588442496 & 0.060588932108 & $4.89611957116 \mathrm{e}-7$ & \\
\hline & & & 0.086998820550 & 0.086999292786 & $4.72236027282 \mathrm{e}-7$ & \\
\hline & & & 0.063576323319 & 0.063576555869 & $2.32549983825 \mathrm{e}-7$ & \\
\hline \multirow[t]{9}{*}{ GSOR } & 1.035 & 11 & 0.337004861075 & 0.337005127298 & $2.66223092793 \mathrm{e}-7$ & 0.00788 \\
\hline & & & 0.485663627469 & 0.485664073550 & $4.46081154737 \mathrm{e}-7$ & \\
\hline & & & 0.381818939520 & 0.381819483734 & $5.44214314557 \mathrm{e}-7$ & \\
\hline & & & 0.155356238509 & 0.155356435644 & $1.97134775309 \mathrm{e}-7$ & \\
\hline & & & 0.223831372917 & 0.223831683168 & $3.10251424107 \mathrm{e}-7$ & \\
\hline & & & 0.167306655006 & 0.167306930693 & $2.75686864226 \mathrm{e}-7$ & \\
\hline & & & 0.060588858201 & 0.060588932108 & $7.39068840572 \mathrm{e}-8$ & \\
\hline & & & 0.086999179535 & 0.086999292786 & $1.13251436340 \mathrm{e}-7$ & \\
\hline & & & 0.063576463795 & 0.063576555870 & $9.20746897754 \mathrm{e}-8$ & \\
\hline
\end{tabular}




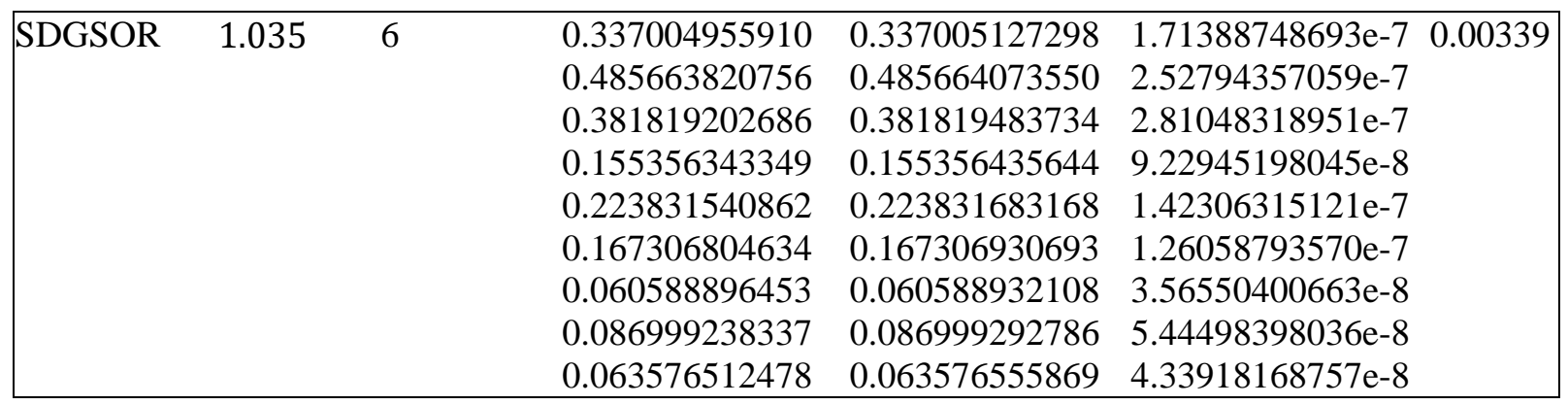

\section{DISCUSSION}

In this paper, a Second Degree Generalized Successive over Relaxation Iterative method for solving large system of Linear Equations is presented. Two practical examples $(4 X 4$, and $9 X 9$ system of linear equations) are considered. The initial approximation for both systems is taken as all zero vectors. The stopping criterion $\left\|x^{(k+1)}-x^{(k)}\right\|<10^{-6}$ is used. We let $\mathrm{m}=1$ and in this case $D_{m}$ is a tri-diagonal matrix. A simple experimental determination of $\omega$ is used to find the optimum relaxation factor. We tried different values of $\omega$ and compared the rates of convergence and continued the experiment with the value of $\omega$ which gives better approximation. The results presented in tables 1, 2, 3 and 4 show that the Second Degree Generalized Successive over relaxation requires less computational running time, less number of iterations and approximates the exact solution better than the other methods used for comparison. Hailu Muleta and Genanew Gofe (2018) stated that method which registers small number of iterations demands less computer storage to store its data. As a result, the Second Degree Generalized Successive over relaxation demands less computer storage to store its data compared to the methods considered for comparison.

\section{ACKNOWLEDGMENTS}

We, the authors, would like to express our heartfelt thanks to Jimma University for funding the paper partially so that it became reality.

\section{CONFLICT OF INTEREST}

There are no conflicts of interest. 


\section{REFERENCE}

Bedet, R.A., Enright, W.H \& Hall, T. E. 1975. STIFF DETEST: A program for comparing numerical methods for stiff Ordinary differential equations. Computing Surveys, 17(1): 2548.

David, R. K. 1994. Stationary second degree iterative methods. Applied Numerical Mathematics, 16(1-2): 227-237.

Davod, S. K. 2007. A Generalization of the SOR method for solving Linear System of Equations. J. Applied Mathematics, Islamic Azad university of Lahijan, 4(15): 31-38

Genanew Gofe. 2016. Refined Iterative Method for Solving System Linear Equations. American Journal of Computational and Applied Mathematics, 16(3): 144-147

Golub, G. H \& Varga, R. S. 1961. Successive Over-Relaxation iterative methods and Second Order Richardson iterative methods. Chebyshev Semi-iterative methods, Numerical mathematics, 3: $147-168$.

Hailu Muleta \& Genanew Gofe. 2018. Refinement of Generalized Accelerated Over Relaxation Method for Solving System of Linear Equations Based on the Nekrassov-Mehmke1Method. Ethiopian Journal of Education and Sciences, 13(2): 1-18.

Kalambi, I. 2008. A Comparison of three Iterative Methods for the Solution of Linear Equations. J. Appl. Sci. Environ. Management, 12(4): 53-55.

Noreen, J. 2012. Comparison of Direct and indirect solvers for linear systems of equations. International Journal of Emerging Sciences, 2(2): 310-321.

Tesfaye Kebede. 2016. Second degree generalized Jacobi iteration method for solving system of linear equations. Tamkang Journal of Mathematics, 47(2):179-192.

Young, D.M. 1972. Second degree iterative methods for the solution of large linear systems. Journal of Approximation Theory, 5: 137-148.

Yang, W. Y., Cao, W., Chung, T \& Morris, J. 2005. Applied Numerical methods using MATLAB. John Wiley \& Sons, INC., ISBN: 9780471698333, Online ISBN: 9780471705192, DOI: 10.1002/0471705195, 509p. 